PHYSICAL REVIEW D 93, 049901(E) (2016)

\title{
Erratum: Full three-body problem in effective-field-theory models of gravity [Phys. Rev. D 90, 084010 (2014)]
}

\author{
Emmanuele Battista and Giampiero Esposito \\ (Received 20 January 2016; published 4 February 2016)
}

DOI: 10.1103/PhysRevD.93.049901

In Eqs. (A9) and (A10), the square roots on the right-hand side should be the square roots of $4 \zeta_{4}^{2}-15 \zeta_{3}$. In Eq. (A11), the factor $(m+\alpha)$ in the denominator should read $(m+\alpha)^{2}$. 